\title{
Electrocardiographic findings in male veteran endurance athletes
}

\author{
ROBIN J NORTHCOTE, GORDON P CANNING, DAVID BALLANTYNE \\ From the Department of Medical Cardiology, Victoria Infirmary, Glasgow
}

SUMMARY Twenty male veteran endurance runners and 20 controls underwent resting, exercise, and ambulatory electrocardiography. Four athletes and three controls satisfied voltage criteria for left ventricular hypertrophy. The PR interval was longer in the athletes and they had longer mean (SD) treadmill exercise times (19 (4) $v 16(2) \mathrm{min}$ ) than the controls. Four athletes but no controls had $>2 \mathrm{~mm}$ downsloping ST segment depression during exercise. During 48 hour ambulatory electrocardiography the athletes had a consistently lower heart rate but maintained a circadian variation. Profound bradycardia ( $<35$ beats $/ \mathrm{min}$ ) occurred in eight athletes but only one control. Eight athletes and two controls had asystolic pauses ranging from 1.8 to 15 seconds. Six athletes had first degree heart block, four had Mobitz II second degree block, and three had complete heart block. Most conduction abnormalities occurred at night and resolved during exercise. Ventricular ectopic activity was not significantly different between the groups.

Thus heart block patterns and profound bradycardia are more frequent in older athletes than their youthful counterparts.

Physicians have recommended habitual long term exercise to all age groups including those with ischaemic heart disease ${ }^{2}$ because of the improvement in general wellbeing, ${ }^{3}$ the plasma lipoprotein profile, ${ }^{45}$ and the reduced risk of developing coronary artery disease. ${ }^{67}$ Although many individuals undertake virtually lifelong endurance exercise, little is known of the long term cardiac effects.

Studies of both resting ${ }^{89}$ and continuous ambulatory electrocardiography ${ }^{10-16}$ have been reported in young athletes. These have shown that bradycardia and heart block patterns are more common in endurance athletes, but with the exception of Pantano and Orieg, who studied some veteran runners (unreported number), ${ }^{11}$ veteran athletes with a long history of habitual exercise have not been studied. Prompted by the referral of several patients with profound bradycardia and heart block patterns who had a history of long term endurance exercise, we studied the resting, exercise, and continuous ambulatory electrocardiographic events in veteran endurance runners and their sedentary controls.

Requests for reprints to Dr Robin J Northcote, Victoria Infirmary, Glasgow G42 9TY.

Accepted for publication 18 August 1988

\section{Patients and methods}

STUDY GROUP

Twenty male veteran endurance runners were recruited from the Scottish Veteran Harriers Club. To satisfy entry criteria all runners had to engage exclusively in running, be over 45 years of age with $>25$ years regular running experience, and run for at least 25 miles per week. The runners were compared with a group of age matched men with a sedentary lifestyle that included some light activity, such as golf, walking, or bowls. None of these men smoked, had any history of cardiovascular disease, or took medication or special diet at the time of study. Table 1 shows the characteristics of both groups.

\section{METHODS}

All subjects underwent resting 12 lead and maximal treadmill exercise test according to a modified Bruce protocol $^{17}$ on a Marquette Case II system. For analysis of the resting electrocardiograms the PR and QT intervals were measured from the mean of ten consecutive beats and the QT interval was corrected for heart rate (QTc) by Bazett's formula. (The upper limit of normal is 0.42 seconds.) The Sokolow and Lyon index ${ }^{18}$ (SV1 + RV5 > $35 \mathrm{~mm}$ ) was used as the criterion for left ventricular hypertrophy. After 
Table 1 Characterisation of study groups (mean (SD))

\begin{tabular}{|c|c|c|c|}
\hline Characteristic & $\begin{array}{l}\text { Endurance } \\
\text { athletes }(n=20)\end{array}$ & $\begin{array}{l}\text { Controls } \\
(n=20)\end{array}$ & pvalue \\
\hline $\begin{array}{l}\text { Age }(y r) \\
\text { Resting heart rate } \\
\text { (beats/min) }\end{array}$ & $\begin{array}{l}56(7) \\
51(7)\end{array}$ & $\begin{array}{l}56(7) \\
72(10)\end{array}$ & $\begin{array}{l}\text { NS } \\
<0.001\end{array}$ \\
\hline $\begin{array}{l}\text { Systolic blood } \\
\text { pressure } \\
\text { (mm Hg) }\end{array}$ & $125(15)$ & $135(23)$ & $<0.01$ \\
\hline $\begin{array}{l}\text { Maximum } \\
\text { treadmill } \\
\text { exercise time } \\
\text { (min) }\end{array}$ & $19(4)$ & $16(2)$ & $<0.01$ \\
\hline $\begin{array}{l}\text { Height (m) } \\
\text { Weight (kg) }\end{array}$ & $\begin{array}{l}1.75(0.048) \\
64(5)\end{array}$ & $\begin{array}{l}1.74(0.079) \\
74(8)\end{array}$ & $\begin{array}{l}\text { NS } \\
<0.01\end{array}$ \\
\hline $\begin{array}{l}\text { Body mass index } \\
\left(\mathrm{Quetelet} \mathrm{kg} /_{\left.\mathrm{cm}^{2} \times 10^{3}\right)}\right.\end{array}$ & $2 \cdot 24(0.01)$ & $2.45(0.25)$ & $<0.01$ \\
\hline$\underset{(y r)}{\text { Running experience }}$ & $36(7)(28-50)$ & - & - \\
\hline Miles/week & $47(23)(25-110)$ & - & - \\
\hline $\begin{array}{l}\text { Best marathon time } \\
\text { (min) }\end{array}$ & $175(23)(2 \cdot 25-3 \cdot 40)$ & - & - \\
\hline
\end{tabular}

at least two hours rest, Holter monitoring was performed with the Medilog 4000 (MARS) ambulatory electrocardiogram recorder (Oxford Medical Systems, Oxford).

This recorder processes each beat/complex in real time and stores the analysed data on a cassette tape. The information was retrieved by the Medilog MARS Replay System (Oxford Medical Systems, Oxford).

After careful skin preparation with an abrasive paste, and shaving if necessary, five electrodes (Medicotest) were applied to the chest wall to produce a recording of lead CM5 and a modified lead II. An impedance meter was then used to ensure that the resistance between each electrode was $<5 \mathrm{k} \Omega$ and surgical tape was used to secure the leads to the chest wall with stress loops to reduce motion artefact. The recorder was worn at the patient's side on a belt. A $1 \mathrm{mV}$ test signal was then recorded and this was used by the system to calibrate analysed data.

Holter monitoring was continued for $\mathbf{4 8}$ hours. For the first 24 hours the patient was instructed to follow his normal daily routine without running or vigorous exercise and to record any symptoms or events in a diary. During the second 24 hours the athletes were asked to run for $\mathbf{4 0}$ minutes at their normal training pace. Before they left the laboratory the electrocardiographic waveform and magnitude being recorded were confirmed on a conventional writer to ensure a satisfactory recording. At the end of each recording the cassette was transferred to the Medilog MARS replay system which generates a comprehensive report via a microdot printer. A full 24 hour recording was obtained for every man and if important abnormalities were found appropriate times were searched at conventional paper speed $(25 \mathrm{~mm} /$
Table 2 Resting electrocardiography

\begin{tabular}{|c|c|c|c|}
\hline & Athletes & Controls & $p$ \\
\hline $\begin{array}{l}\text { SV1 + RV5 } \\
\text { PR } \\
\text { QTc } \\
\text { QRS }\end{array}$ & $\begin{array}{l}28(7.2) \\
0.20(0.04) \\
0.42(0.027) \\
0.10(0.01)\end{array}$ & $\begin{array}{l}25(7.8) \\
0.18(0.03) \\
0.42(0.027) \\
0.10(0.01)\end{array}$ & $\begin{array}{l}<0.05 \\
<0.05 \\
\text { NS } \\
\text { NS }\end{array}$ \\
\hline
\end{tabular}

$\mathrm{PR}, \mathrm{QRS}$ intervals, and QTc derivative measured in seconds. All data given are mean (SD).

s). Mean nocturnal heart rate was derived from the ${ }_{\infty}^{\omega}$ mean of the hourly rates between midnight and 6 am? when the men were in bed. Maximum and minimumos heart rates were recorded from the average $\mathbf{R R}_{\dot{\mathrm{j}}}^{-}$ interval over four complexes. An asystolic pause wasdefined as asystole lasting $>1.5$ seconds and $1.9 \mathrm{G}$ times longer than the average RR interval of the fouro previous complexes. Ventricular tachycardia wasregarded as being present if three or more con-T secutive premature ventricular contractions은 occurred at a rate exceeding 110 beats $/ \mathrm{min}$. If the rate was less than 110 beats/min then idioventricular rhythm was regarded as being present. Premature $\vec{\bullet}$ ventricular contractions were classified according too the Lown grading system. ${ }^{19}$

\section{Results}

\section{RESTING ELECTROCARDIOGRAMS}

At rest two athletes had evidence of both second (Mobitz type II) and third degree heart block (QRSS duration 0.08 and $0.10 \mathrm{~s}$ respectively). Four athletes, and three controls satisfied voltage criteria for lefo ventricular hypertrophy, the athletes having a greater mean voltage than the controls (table 2). There was a significantly prolonged $P R$ interval in? the athletes $(p<0.05)$ but no difference in the QTc? derivative or $\mathrm{QRS}$ duration.

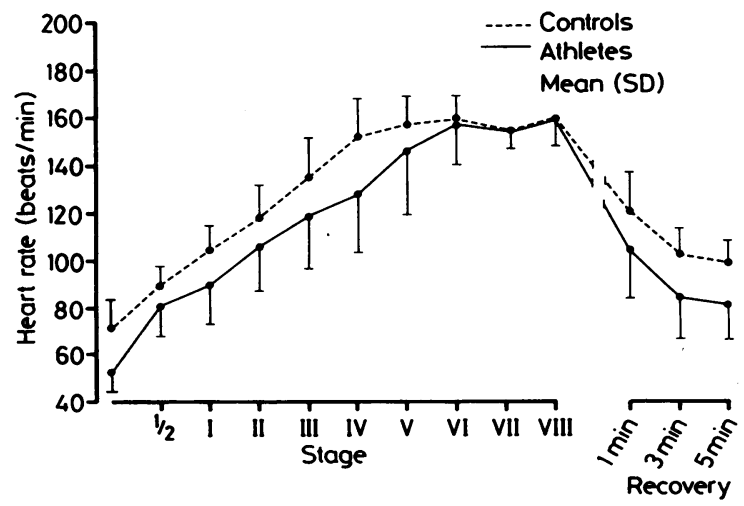

Fig 1 Heart rate response to treadmill exercise test and in the immediate post-exercise recovery period. 


\section{EXERCISE ELECTROCARDIOGRAPHY}

The mean (SD) maximum treadmill exercise time was 19 (4) minutes in athletes and 16 (2) minutes in the control group ( $p<0.01$ ). As a group the athletes were able to perform more work at a lower heart rate. Twelve athletes but only four controls surpassed stage $\mathrm{V}$ of the protocol. Figure 1 shows the heart rate responses to treadmill exercise and fig 2 the exercise times. Four athletes but no controls had $>2 \mathrm{~mm}$ of downsloping ST segment depression during exercise and one other had frequent unifocal premature ventricular complexes after exercise. Two athletes who developed ST segment depression were offered coronary angiography, one because of the subsequent development of chest pain and one because of the concurrent finding of complete heart block with profound bradycardia ( $<25$ beats $/ \mathrm{min}$ ). Both arteriograms were entirely normal. (Figure 3 shows the exercise electrocardiograms.)

\section{AMBULATORY ELECTROCARDIOGRAPHY}

The athletes had a consistently lower heart rate at all times of the day (table 3). Eight athletes but only one control had a profound sinus bradycardia $(<35$ beats/min); the lowest heart rate in an athlete was 17 beats/min recorded at 3 am while he was asleep. The circadian variation of heart rate was maintained in athletes despite the bradycardia (fig 4). Eight athletes and two controls had evidence of asystolic pauses $(>1.5 \mathrm{~s}$ ); one of them had 846 episodes. This individual had pauses ranging from 1.8 to 15 seconds and had evidence of first, second (2:1 Mobitz type II), and third degree atrioventricular block at various times of the day. This individual was not prepared to stop his endurance running and because we believed that he might be at risk of cerebrovascular accident ${ }^{20}$ or even sudden death we decided to implant a

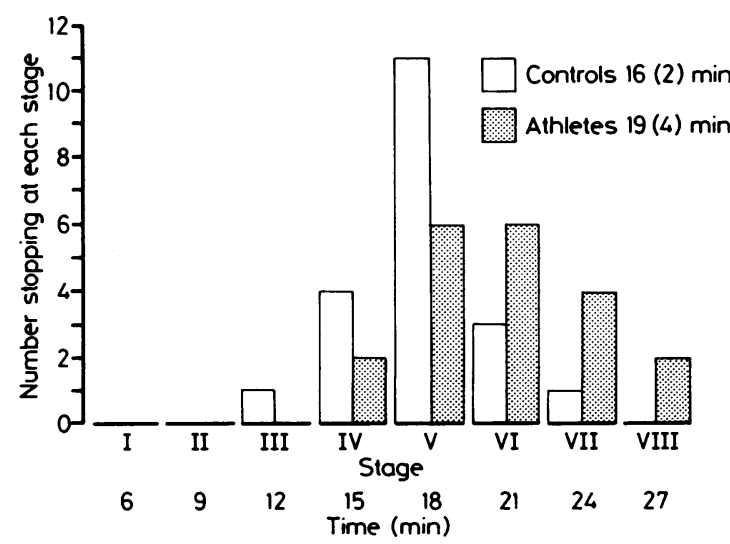

Fig 2 Exercise times on treadmill according to the stage at which an individual stopped because of fatigue or breathlessness.
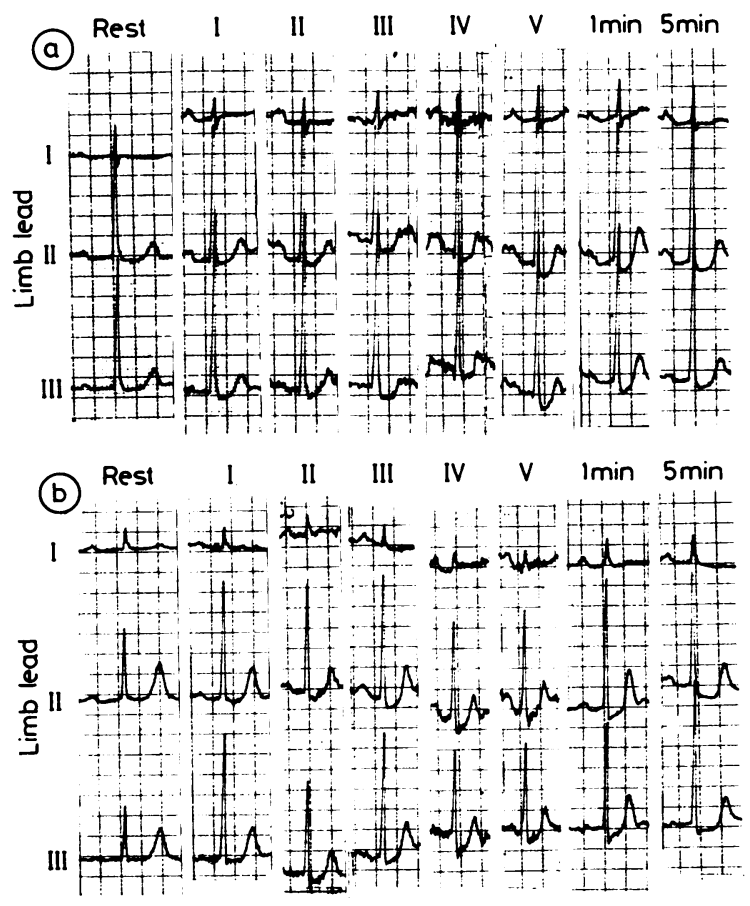

Fig 3 Exercise electrocardiograms of two of the four athletes who had an "ischaemic response" and who were offered coronary arteriography. The electrocardiograms show a progression through the stages of a Bruce protocol and the immediate post-exercise recovery period.

permanent dual chamber pacemaker (Pacesetter AFP 283). Interestingly, although the patient did not report any symptoms before implantation, he remarked that he felt more energetic afterwards and was able to reduce his time for a 10 mile run by several minutes. Six athletes had a prolonged PR interval ( $>0.22 \mathrm{~s}$ ), four had second degree atrioventricular block, and three had complete or third degree heart block. Apart from the two men who had four episodes of asystole between them, no conduction abnormalities were found in the control group. Most asystolic pauses occurred at night (fig 5).

Two of the athletes with complete heart block also had an abnormal electrocardiographic response to

Table 3 Heart rates derived from continuous 24 hour electrocardiography (mean $(S D)$ )

\begin{tabular}{lccl}
\hline Heart rate & Athletes & Controls & $p$ \\
\hline Mean 24 hour & $59(8.3)$ & $74(9.3)$ & $<0.001$ \\
Minimum & $37(7.8)$ & $47(6.0)$ & $<0.01$ \\
Maximum & $121(21.9)$ & $144(18.6)$ & \\
Mean daytime & $61(8.2)$ & $76(9.5)$ & $<0.001$ \\
Mean nocturnal & $51(8.5)$ & $66(13.2)$ & $<0.001$ \\
\hline
\end{tabular}




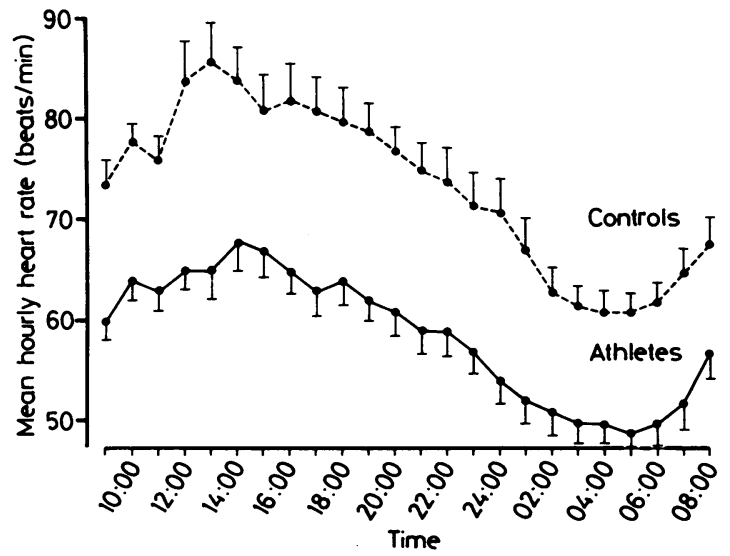

Fig 4 Twenty four hour trend of heart rate in athletes and controls (mean hourly rate ( $S E)$ ).

treadmill exercise. As previously mentioned one of them underwent coronary angiography with a normal result. During sedentary activities 15 of the 20 athletes had at least one premature ventricular contraction per 24 hours compared with 18 of the controls, but only four athletes and four controls had $>50$ premature ventricular contractions per 24 hours. One athlete had 1652 in 24 hours. This man had multifocal extrasystoles normally distributed throughout 24 hours including couplets and a short episode of ventricular trigeminy. In addition he also had a short episode of paroxysmal atrial tachycardia. When his data were excluded, there was no significant difference between the total number of premature ventricular contractions occurring in both groups (380 v 370) (table 4).

Two controls had episodes of self terminating ventricular tachycardia, one of 18 and the other of 60 complexes. The premature ventricular contractions

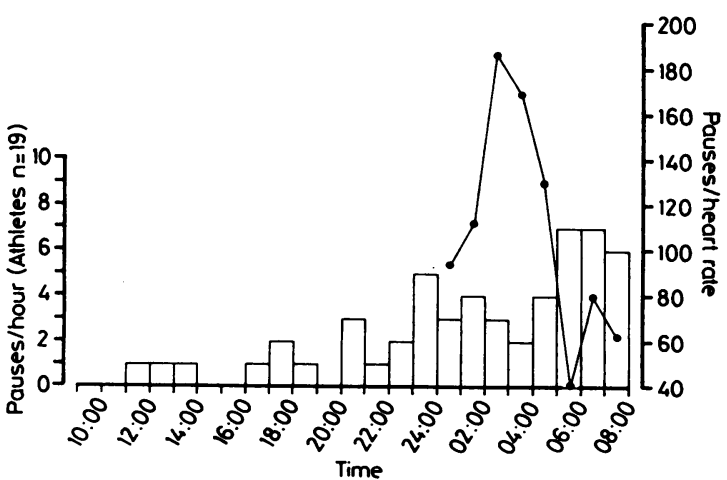

Fig 5 Frequency distribution of asystolic pauses throughout 24 hours. Solid line shows the distribution in a man with 846 pauses in 24 hours, which only occurred between the hours of 0100 and 0800.
Table 4 Classification of ventricular ectopic activity in athletes and controls

\begin{tabular}{|c|c|c|c|c|}
\hline \multirow[b]{2}{*}{ Lown grading } & \multicolumn{2}{|c|}{ Athletes } & \multicolumn{2}{|c|}{ Controls } \\
\hline & No & $\begin{array}{l}\% \text { of } \\
\text { total }\end{array}$ & No & \\
\hline 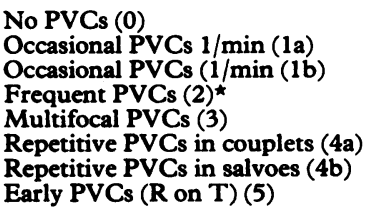 & $\begin{array}{r}5 \\
12 \\
1 \\
2 \\
3 \\
2 \\
0 \\
1\end{array}$ & $\begin{array}{r}25 \\
60 \\
5 \\
10 \\
15 \\
10 \\
0 \\
5\end{array}$ & $\begin{array}{r}2 \\
18 \\
0 \\
2 \\
1 \\
1 \\
2 \\
0\end{array}$ & $\begin{array}{rl}10 & \\
90 & \\
0 & \vdots \\
10 & \vdots \\
5 & 3 \\
5 & \\
10 & \vdots \\
0 & \end{array}$ \\
\hline
\end{tabular}

* Ventricular bigeminy and trigeminy are grouped under Lown grade 2.

PVC, premature ventricular contractions.

were mostly unifocal and occurred sporadically ${ }^{\circ}$ throughout 24 hours (fig 6). During the second $\overrightarrow{\text { Th }}$ period of 24 hours, in which the athletes completed ao 40 minute road run, 11 of 17 athletes (the recordings? on three athletes were unsuitable for analysis because of electrode displacement) had scattered premature ventricular contractions; most of these were isolatedoo and unifocal (Lown grade 1a in ten cases and Lown grade $1 b$ in one case). One individual had evidence of $R$ on $T(R R / Q T<0.85)$ during running (Lown grade 5) but, in general, training did not appear to provoke an increased frequency of ventricularo extrasystoles. The maximum heart rate achieved $\mathbb{Q}$ during outdoor running was 162 (18) beats/min $\overrightarrow{0}$ (range 122-196), equivalent to a predicted maximum 3 heart rate for the group of $99 \%$. All patterns of atrioventricular block disappeared during exercise and there was no correlation between the presence of premature ventricular contractions and an abnormale electrocardiographic response to treadmill exercise.

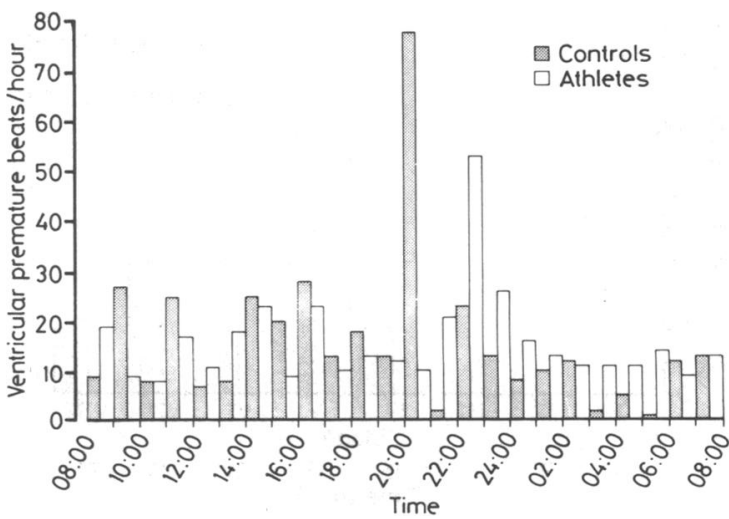

Fig 6 Frequency histogram of PVCs in athletes and controls throughout 24 hours (excluding athletes with $>1600$ 을 PVCs per 24 hours). 


\section{Discussion}

We compared athletes with a group of healthy control subjects who participated in light physical activity. All the athletes trained exclusively by distance running and did not use weight training or resistance exercise; thus any confusion with the effects of anaerobic or power exercise (including isometric exercise) was avoided. The controls were chosen from a group of volunteers. Clearly, habitual long distance runners -are very difficult to match with a control population. They tend to be very lean, from high educational and socioeconomic groups, and are conscious of their diet and health. The control group in this study was not an inactive slothful cohort of middle aged men and this is reflected in the mean treadmill exercise time of 16 minutes. So we believe that our control group was as closely matched as possible.

Athlete's bradycardia is a well documented phenomenon and has been investigated in young athletes by several groups. ${ }^{10-15}$ Like us, Viitsalo et al found that despite the bradycardia, circadian variation of heart rate was maintained. ${ }^{14}$ The cause of bradycardia in athletic subjects is uncertain. Most evidence suggests that the sinoatrial node and atrioventricular nodes are suppressed by an increase in vagal tone, which can be abolished by atropine or stopping training. ${ }^{821}$ However, increased vagal tone may only be partly responsible because studies of surgical denervation and pharmacological denervation (atropine plus propranolol) have shown the bradycardia to be independent of vagal tone. ${ }^{22} 23$ Thus the intrinsic heart rate is lower.

Although the bradycardia seen in athletes is currently recognised as a benign adaptation, it has been associated with unexplained cerebrovascular accidents $^{20}$ and becomes more notable when associated with atrioventricular block patterns. It is possible that continuous habitual vigorous exercise throughout life may result in a bradycardia which, rather than being adaptive, has been modified into a condition resembling the sick sinus syndrome. It is for this reason that one of the subjects in the present study, who also had prolonged periods of asystole, had implantation of a permanent pacemaker. Until follow up studies of patients with exercise induced bradycardia and atrioventricular block have been published, cardiologists will have no firm guidelines on the management of this condition. If an athlete does not wish to stop exercising, then pacemaker implantation may need to be considered in severe cases, even in the absence of symptoms.

Several studies have noted the presence of asystolic pauses in athletic populations. ${ }^{19121416}$ Typically these have lasted from 1.6 to $2 \cdot 8 \mathrm{~s}$. We have been unable to find any other reports of pauses of up to 15 seconds in the absence of symptoms. Ector et al studied 16 sportsmen who presented with syncope. ${ }^{16}$ Half of them became symptom free on stopping training, although another seven required implantation of a permanent pacemaker. Thus stopping training does not always resolve the problem. Although first degree and, to a lesser extent, second degree atrioventricular block has been found in younger athletes $^{10121415}$ complete heart block in a symptom free normal or athletic population is exceedingly rare. In athletes the frequency of first degree heart block varies from $5 \%$ to $55 \%^{812} 1415$ but is typically around $20 \%$. Second degree atrioventricular block (Mobitz type II and Wenckebach) is less common and is found in $13-22 \%$ of athletes. ${ }^{1214-16}$ Thus the frequency of first and second degree atrioventricular block (Mobitz type II) found in this study is similar to that in younger athletes but none of the earlier studies reported complete heart block, which occurred in three of our athletes. ${ }^{10-15}$ The occurrence of block patterns in athletes is probably related to increased vagal tone, because resolution can occur during exercise. However, in some veteran athletes it may coexist with the sick sinus syndrome and thus lead to profound bradycardia and symptoms. The resting electrocardiograms of the athletes in this study were unhelpful in identifying all those with atrioventricular block, although complete heart block was found in two men.

In contrast with other sports, which exert a predominantly pressure overload on the left ventricle, running induces volume overload. Thus only four of 20 athletes $(20 \%)(\mathrm{S} 1+\mathrm{R} 5$ mean $=28 \mathrm{~mm})$ satisfied voltage criteria for left ventricular hypertrophy compared with 101 (35\%) of 289 professional American Football players trained on power and resistance exercise ${ }^{9}(\mathrm{~S} 1+\mathrm{R} 5$ mean $=37 \mathrm{~mm})$. Although the QTc derivative may be prolonged in some athletes ${ }^{24} 25$ we did not find any difference between our runners and controls.

Four athletes in this study had an exercise electrocardiogram that satisfied the criteria for ischaemia (greater than $1 \mathrm{~mm}$ downward sloping ST segment depression occurring 0.08 seconds after the $\mathrm{J}$ point)-two of them had entirely normal coronary arteriograms. This is more than would be expected in a normal symptom free population. Balady et al found that $13 \%$ of power trained athletes had ST segment depression on resting electrocardiograms ${ }^{9}$ and Pantano and Orieg describes three well trained runners who had an ischaemic response to treadmill exercise but who had normal thallium-201 myocardial perfusion scans. ${ }^{11}$ The reason for this false positive response is not clear. It has been suggested that athletes' ventricles repolarise in a non-homo- 
160

geneous fashion with repolarisation of the epicardium occurring first. ${ }^{26}$ The asymmetry of repolarisation may be related to reduced sympathetic tone. ${ }^{27}$ Although two previous studies in healthy middle aged men suggested that long term participation in exercise results in fewer premature ventricular contractions, ${ }^{28} 29$ there was no difference between the groups in the frequency of premature ventricular contractions in this study. The frequency (only $20 \%$ in each group had $>50$ premature ventricular contractions per 24 hours) was similar to that reported in younger athletes ${ }^{101214}$ and unlike Pantano and Orieg ${ }^{11}$ we did not find that the frequency of premature ventricular contractions was increased during out of hospital exercise training. In the symptom free athlete it is highly probable that infrequent premature ventricular contractions are a normal finding that should not precipitate further investigation.

\section{References}

1 Ehsani AA, Martin WH, Heath GW, Coyle EF. Cardiac effects of prolonged and intense exercise training in patients with coronary artery disease. Am J Cardiol 1982;50:246-54.

2 Redwood DR, Rosing DR, Epstein SE. Circulatory and symptomatic effects of physical training in patients with coronary artery disease and angina pectoris. N Engl J Med 1972;286:959-65.

3 Clark RS, Ballantyne D. Physical activity and coronary heart disease. Scott Med J 1981;26:15-20

4 Ballantyne FC, Clark RS, Simpson HS, Ballantyne D. The effect of moderate physical exercise on the plasma lipoprotein subfractions of male survivors of myocardial infarction. Circulation 1982;65:913-8.

5 Northcote FJ, Todd IC, Canning GP, Ballantyne D. Lipoprotein profiles of elite veteran endurance athletes. Am J Cardiol 1988;61:934-6.

6 Paffenbarger RS, Hyde RT. Exercise as protection against heart attack. $N$ Engl J Med 1980;302:1026-7.

7 Eichner ER. Exercise and heart disease: epidemiology of the "exercise hypothesis". Am J Med 1983; 75:1008-23.

8 Lichtman J, O'Rourke RA, Klein A, Karliner JS. Electrocardiogram of the athlete. Arch Intern Med 1973;132:763-70.

9 Balady GJ, Cadigan JB, Ryan TJ. Electrocardiogram of the athlete: an analysis of 289 professional football players. Am J Cardiol 1984;53:1339-43.

10 Talan DA, Bauernfeind RA, Ashley WW, Kanakis C, Rosen KM. Twenty-four hour continuous ECG recordings in long distance runners. Chest 1982;1: 19-24.

11 Pantano JA, Orieg RG. Prevalence and nature of cardiac arrhythmias in apparently normal well trained runners. Am J Heart J 1982:104:762-8.

12 Viitasalo MT, Kala R, Eisalo A. Ambulatory electrocar-
Northcote, Canning, Ballantyne $\frac{\text { Ф }}{\frac{1}{\Phi}}$

diographic recording in endurance athletes. $\mathrm{Br}$ Heart. $J$ 1982;47:213-20.

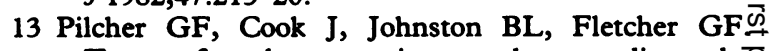
Twenty-four hour continuous electrocardiography? during exercise and free activity in 80 apparentlyo healthy runners. Am J Cardiol 1983;52:859-61.

14 Viitasalo MT, Kala R, Eisalo A. Ambulatory electrocardiographic findings in young athletes between 14-16 years of age. Eur Heart $J$ 1984;5:2-6.

15 Palatini $P$, Marablino G, Sperti G, et al. Prevalence and possible mechanisms of ventricular arrhythmias inathletes. Am Heart J 1985;110:560-7.

16 Ector H, Verlinden M, Vanden Eynde E, et al $\stackrel{\odot}{\circ}$ Bradycardia, ventricular pauses, syncope and sports Lancet 1984;ii:591-4.

17 Bruce RA, Gey GO, Cooler MN, Fisher LD, Patersorn DR. Seattle Heart Watch: initial clinical, circulatoryand electrocardiographic response to maximal exer $\mathrm{N}$ cise. Am J Cardiol 1974;33:459-69.

18 Sokolow M, Lyon TP. The ventricular complex in left ventricular hypertrophy as obtained by unipolar $T$ precordial and limb leads. Am Heart $J$ 1949;37 $\mathbb{O}$ 161-86.

19 Lown R, Wolf MA. Approaches to sudden death from coronary artery disease. Circulation 1971;44:130-42.

20 Abdon N-J, Landin K, Johansson BW. Athlete's $\overrightarrow{0}$ bradycardia as an embolising disorder? Symptomatice arrhythmias in patients aged less than 50 years. $B$ ir Heart J 1984;52:660-6.

21 Meytes I, Kaplinsky E, Yahinni J, Hanne-Paparo N, Neufeld HN. Wenckebach AV block: a frequen feature following heavy physical training. Am Heart 1975;90:426-30.

22 Badeer HS. Cardiovascular adaptations in the trained athlete. In: Lubich T, Venerando A, eds. Sportş cardiology. Bologna: Aulo Caggi, 1980:3-12.

23 Ordway GA, Charles JR, Randall DC, Billman GE Wekstein DR. Heart rate adaptation to exercise training in cardiac denervated dogs. J Appl Physio 1982;52:1586-90.

24 Beswick FW, Jordan RC. Cardiological observations atithe sixth British Empire and Commonwealth Games? Br Heart J 1961;23:113-30.

25 Van Ganse W, Versee L, Eylenbosch W, Vuylsteek $K \stackrel{\circ}{2}$ The electrocardiogram of athletes: comparison with untrained subjects. Br Heart $J$ 1970;32:160-4.

26 Kambara H, Phillips J. Long-term evaluation of early repolarisation syndrome (normal variant RS-T segment elevation). Am J Cardiol 1976;38:157-61.

27 Zeppilli P, Pirrami MM, Sassara M, Fenici R. T wave abnormalities in top ranking athletes: effects of isoproterenol, atropine and physical exercise. $A m$ U Heart J 1980;100:213-22.

28 Blackburn $\mathrm{H}$, Taylor $\mathrm{HL}$, Hamrell B, Buskirk $\mathrm{E}_{\mathrm{O}}^{2}$ Nicholas WC, Thorsen RD. Premature ventricular complexes induced by stress testing, their frequencyes and response to physical conditioning. Am J Cardiol 1973;31:441-9.

29 Viitasalo MT, Kala R, Eisalo A, Halonen PI. Ven tricular arrhythmias during exercise testing, jogging and sedentary life. Chest 1979;76:21-6. 